\title{
A Finite-Time Disturbance Observer Based Full-Order Terminal Sliding-Mode Controller for Manned Submersible with Disturbances
}

\author{
Xing Fang $(\mathbb{D})$ and Fei Liu \\ Key Laboratory of Advanced Process Control for Light Industry (Ministry of Education), Institute of Automation, \\ Jiangnan University, Wuxi 214122, China \\ Correspondence should be addressed to Xing Fang; xingfang@jiangnan.edu.cn
}

Received 15 June 2018; Revised 10 September 2018; Accepted 16 September 2018; Published 30 September 2018

Academic Editor: Luis J. Yebra

Copyright ( 2018 Xing Fang and Fei Liu. This is an open access article distributed under the Creative Commons Attribution License, which permits unrestricted use, distribution, and reproduction in any medium, provided the original work is properly cited.

\begin{abstract}
A novel full-order terminal sliding-mode controller (FOTSMC) based on the finite-time disturbance observer (FTDO) is proposed for the "JIAOLONG" manned submersible with lumped disturbances. First, a finite-time disturbance observer (FTDO) is developed to estimate the lumped disturbances including the external disturbances and model uncertainties. Second, a full-order terminal sliding-mode surface is designed for the manned submersible, whose sliding-mode motion behaves as full-order dynamics rather than reduced-order dynamics in conventional sliding-mode control systems. Then, a continuous sliding-mode control law is developed to avoid chattering phenomenon, as well as to drive the system outputs to the desired reference trajectory in finite time. Furthermore, the closed-loop system stability analysis is given by Lyapunov theory. Finally, the simulation results demonstrate the satisfactory tracking performance and excellent disturbance rejection capability of the proposed finite-time disturbance observer based full-order terminal sliding-mode control (FTDO-FOTSMC) method.
\end{abstract}

\section{Introduction}

Manned submersibles have always been indispensable equipment for navy and marine development. Recently, there are great many manned submersibles that are on active service around the world [1], such as Alvin (USA), Shinkai 6500 (Japan), and so on. China's manned submersible named "JIAOLONG" is designed for deep-sea exploration [2, 3]. JIAOLONG carried out its $7000 \mathrm{~m}$ exploration cruise in June 2012 , which implies that JIAOLONG is able to cover $99.8 \%$ of the ocean area all over the world.

Manned submersibles always work in complicated ocean environment, including large water pressure in deep sea, time-varying ocean currents, unpredictable external disturbances, unknown obstacles, and so on $[4,5]$. Furthermore, manned submersibles are known as strong nonlinear systems with various disturbances and time-varying dynamics $[6,7]$. To the best of the authors' knowledge, there are only few published papers regarding the robust controller design for manned submersibles, especially to attenuate the various disturbances. Therefore, it is an open challenging research area to investigate the high-performance controller for manned submersibles with disturbances.

In the past decades, some attempts have been made to handle the control problem of manned submersibles. A robust controller based on $H_{\infty}$ algorithm is developed for the manned submersible to suppress the external disturbances [8]. However, the control law is designed based on the worst case, which will result in overconservative control performance. Adaptive control techniques have been employed to deal with parameter uncertainties of the manned submersibles [9]. Unfortunately, the adaptive control may fail to handle the parameter uncertainties when the parameter changing speed is beyond its adapting capability. With the unique disturbance attenuation capability, slidingmode control (SMC) methods have been employed to suppress the external disturbances and model uncertainties of manned submersibles [10-13]. However, the conventional SMC method will bring about chattering phenomenon for 
its discontinuous control action [14]. Thus, some attempts have been made to alleviate the chattering phenomenon [15-17]. To sum up, the aforementioned control methods deal with the external disturbances and model uncertainties with the manner of feedback control action indirectly and slowly, rather than the feed-forward control action that is able to compensate the effects of the disturbances and uncertainties directly. Therefore, feedback control methods may fail to reject the severe disturbances and uncertainties of the manned submersible systems.

In recent years, the disturbance-observer-based control (DOBC) methods have been proposed to deal with the disturbances and uncertainties widely [18-20]. The DOBC technique is regarded as a two-degree-of-freedom control method, which consists of a nominal controller and a disturbance rejector [21]. The nominal controller is developed to achieve the requirements on tracking performance specifications and stability of the system. On the other hand, the disturbance rejector will be designed to meet the requirements on disturbance rejection and robustness for the system. That is to say, these two (often conflicting) requirements can be satisfied by developing the nominal feedback controller and disturbance rejector separately [22]. Furthermore, the DOBC methods have been applied to a wide range of engineering systems, such as permanent magnet synchronous motor (PMSM) system [23], unmanned helicopter system [24], power plant system [25], and so on.

Among the large number of DOBC methods, the combination of sliding-mode control technique and disturbance observer has drawn much attention of the scholars, which is called as disturbance-observer based sliding-mode control (DOB-SMC) method. For the reason that the SMC techniques not only have the powerful disturbance-attenuation capability, but also possess the simple design procedure of the controller. In the past few years, a nonlinear disturbance observer based sliding-mode control is employed to attenuate the external disturbances and model uncertainties, which is applied to MAGLEV suspension system [26]. In addition, a new disturbance observer based terminal sliding mode control method is designed for multiple-input multipleoutput (MIMO) nonlinear system, which is able to suppress both the disturbances generated by an exogenous system and the disturbances with bounded $H_{2}$ norm [27]. The article [28] develops an extended disturbance observer based slidingmode control to reject both the matched and mismatched disturbances of the unmanned helicopter systems. Recently, an extended state observer based integral sliding-mode control method has been employed to attenuate the disturbances and uncertainties of the underwater robot system [29]. Although the aforementioned DOB-SMC methods are able to attenuate the disturbances effectively, the chattering phenomenon exists in the system all the same because of the discontinuous control actions. Furthermore, all the existing DOBSMC methods belong to reduced-order sliding-mode control techniques, which means that the sliding-mode motion of the system behaves as desirable reduced-order dynamics rather than desirable full-order dynamics. Recently, a fullorder sliding-mode control (FOSMC) algorithm is designed in article [30]. However, the sliding variable of the proposed
FOSMC algorithm is not available directly, which brings about the difficulties for controller implementation. Additionally, this FOSMC method attenuates the disturbances by the means of feedback control technique indirectly. Therefore, the disturbance rejection capability needs to be further improved.

In this paper, a novel finite-time disturbance observer based full-order terminal sliding-mode control (FTDOFOTSMC) method is developed for the manned submersible subject to external disturbances and model uncertainties. By designing the full-order terminal sliding-mode surface, the ideal sliding-mode motion of the manned submersible system behaves as desirable full-order dynamics. Furthermore, with the estimation of the disturbances by FTDO, the continuous sliding-mode control law is designed to compensate the lumped disturbances of manned submersible and to meet the requirements on tracking performance. Finally, both the rigorous theoretical analysis and simulation results are presented to verify the superiority of the proposed FTDO-FOTSMC method.

The most outstanding novelties of this paper are highlighted as follows. First of all, under the proposed novel FTDO-FOTSMC method, the ideal sliding-mode motion of the system behaves as desirable full-order dynamics rather than reduced-order dynamics. Additionally, the FTDOFOTSMC method is able not only to reject the external disturbances and model uncertainties, but also to eliminate the chattering phenomenon in conventional sliding-mode control systems. Furthermore, the FTDO-FOTSMC method is applied to the "JIAOLONG" manned submersible system, which achieves satisfactory control performance.

This paper is organized as follows. Section 2 analyzes the manned submersible system. A novel FTDO-FOTSMC method is proposed for the manned submersible system to reject the lumped disturbances in Section 3. The stability analysis and simulation results are presented in Sections 4 and 5, respectively. Finally, some conclusions are drawn in Section 6.

\section{Manned Submersible Model}

This section presents the nonlinear dynamic model of the manned submersible. The manned submersible is considered as a six-degree-of-freedom rigid body model [6]. Furthermore, it is subject to external disturbances and model uncertainties, which is treated as the lumped disturbances.

To develop the model of manned submersible, two reference frames, i.e., the navigation frame $F_{n}=\left\{O_{n}, i_{n}, j_{n}, k_{n}\right\}$ and body frame $F_{b}=\left\{O_{b}, i_{b}, j_{b}, k_{b}\right\}$, are defined, which are shown in Figure 1. [7]:

The nonlinear model can be presented by the following

$$
\begin{aligned}
\dot{\eta} & =J(\eta) v, \\
M \dot{v}+C(v) v+D(v) v+G(\eta) & =F+d,
\end{aligned}
$$

where $\eta=\left[\begin{array}{ll}\eta_{1}^{T} & \eta_{2}^{T}\end{array}\right]^{T} \in R^{6}$ denotes the position and Euler angle vector in the navigation frame, and $v=\left[\begin{array}{ll}v_{1}^{T} & v_{2}^{T}\end{array}\right]^{T} \in R^{6}$ 

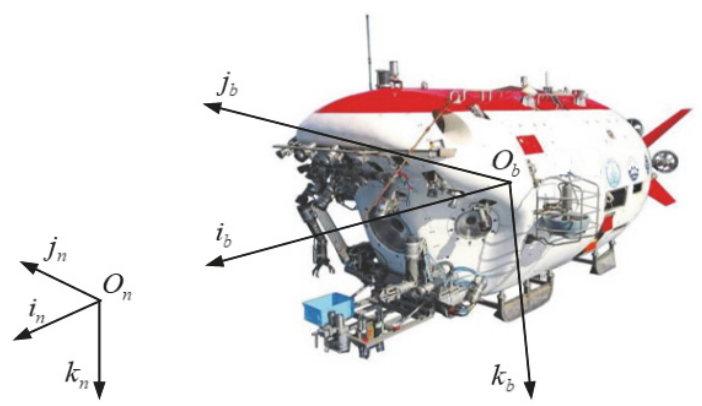

FIGURE 1: Navigation and body frames of manned submersible.

denotes the linear velocity and angular velocity vector in the body frame. Furthermore, $\eta_{1}=\left[\begin{array}{lll}x & y & z\end{array}\right]^{T}, \eta_{2}=\left[\begin{array}{lll}\varphi & \theta & \psi\end{array}\right]^{T}$, $v_{1}=\left[\begin{array}{lll}u & v & w\end{array}\right]^{T}$ and $v_{2}=\left[\begin{array}{lll}p & q & r\end{array}\right]^{T}$. The notations $J(\eta) \epsilon$ $R^{6 \times 6}, M \in R^{6 \times 6}, C(v) \in R^{6 \times 6}, D(v) \in R^{6 \times 6}$ and $G(\eta) \in R^{6}$ represent the rotation matrix, inertia matrix, Coriolis and centrifugal matrix, hydrodynamic damping matrix, and the gravity and buoyancy forces, respectively. $F \in R^{6}$ represents the control forces and moments acting on the manned submersible, and $d \in R^{6}$ denotes the external disturbances.

In practical engineering applications, it is difficult to acquire the accurate hydrodynamics coefficients of the manned submersible. Therefore, the hydrodynamics coefficients can be divided into two parts: nominal value and bias value. $M=M_{0}+\Delta M, C(v)=C_{0}(v)+\Delta C(v), D(v)=$ $D_{0}(v)+\Delta D(v)$ and $G(\eta)=G_{0}(\eta)+\Delta G(\eta)$, where $(\cdot)_{0}$ denotes the nominal value, and $\Delta(\cdot)$ denotes the bias value.

On the other hand, the manned submersible equips seven thrusters and one trim adjusting mercury pump, which are the main power sources of the submersible. Thus, the control forces and moments can be described by

$$
F=B u,
$$

where $u \in R^{8}$ is the true control input vector, and $B \in R^{6 \times 8}$ is the force allocation matrix.

Considering the factors mentioned above, the dynamics equation (2) of the manned submersible can be rewritten by

$$
M_{0} \dot{v}+C_{0}(v) v+D_{0}(v) v+G_{0}(\eta)=B u+d_{s},
$$

where $d_{s}$ presents the lumped disturbances containing the external disturbances and model uncertainties, which can be described by

$$
d_{s}=d-\Delta M \dot{v}-\Delta C(v) v-\Delta D(v) v-\Delta G(\eta) .
$$

Furthermore, for the convenience of controller design, the complete nonlinear model of the manned submersible can be written by

$$
\begin{aligned}
\dot{\eta}= & v_{a} \\
\dot{v}_{a}= & -\bar{C}(\eta, v) v_{a}-\bar{D}(\eta, v) v_{a}-\bar{G}(\eta, v)+\bar{M}(\eta, v) B u \\
& +d_{l},
\end{aligned}
$$

where $v_{a}=J(\eta) v$ is the new state vector, $d_{l}=\bar{M}(\eta, v) d_{s}$ is the new lumped disturbances. $\bar{C}(\eta, v)=J(\eta) M_{0}^{-1} C_{0}(v) J^{-1}(\eta)-$

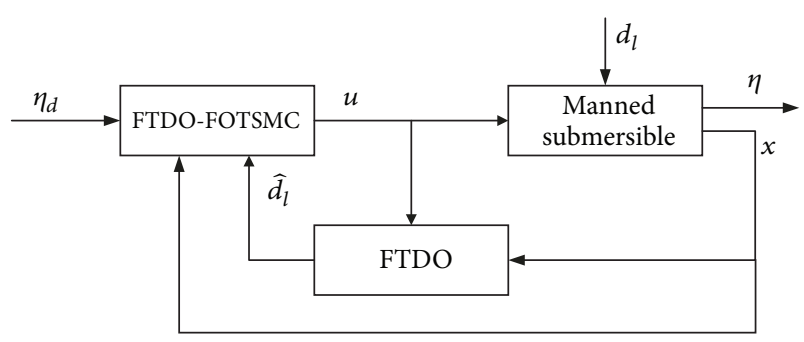

FiguRE 2: The controller structure of manned submersible.

$\dot{J}(\eta) J^{-1}(\eta), \bar{D}(\eta, v)=J(\eta) M_{0}^{-1} D_{0}(v) J^{-1}(\eta), \bar{G}(\eta, v)=$ $J(\eta) M_{0}^{-1} G(\eta)$ and $\bar{M}(\eta, v)=J(\eta) M_{0}^{-1}$. Additionally, for the sake of convenience in writing, the notations $\bar{C}, \bar{D}, \bar{G}$ and $\bar{M}$ are used to replace $\bar{C}(\eta, v), \bar{D}(\eta, v), \bar{G}(\eta, v)$, and $\bar{M}(\eta, v)$ in this paper.

\section{Controller Design}

In this section, a novel finite-time disturbance observer based full-order terminal sliding mode controller (FTDOFOTSMC) is designed for the manned submersible system. The controller is able to drive the position and Euler angle of the manned submersible to track the desired reference trajectory of $\eta_{d}=\left[\begin{array}{llllll}x_{d} & y_{d} & z_{d} & \varphi_{d} & \theta_{d} & \psi_{d}\end{array}\right]^{T}$ despite the presence of lumped disturbances $d_{l}$. The composite controller structure of manned submersible is illustrated in Figure 2.

3.1. Finite-Time Disturbance Observer (FTDO). A finite-time disturbance observer (FTDO) will be developed to estimate the lumped disturbances $d_{l}$ of the manned submersible in finite time.

To design the FTDO conveniently, the dynamics model (7) of manned submersible is rewritten by

$$
\dot{v}_{a}=f\left(\eta, v_{a}, u\right)+d_{l},
$$

where $f\left(\eta, v_{a}, u\right)=-\bar{C}(\eta, v) v_{a}-\bar{D}(\eta, v) v_{a}-\bar{G}(\eta, v)+$ $\bar{M}(\eta, v) B u \in R^{6}$ is the vector function. Additionally, the notation $f$ is used to replace $f\left(\eta, v_{a}, u\right)$.

A first-order FTDO is designed to estimate the lumped disturbances $d_{l}$ as follows [31]:

$$
\begin{aligned}
\dot{\xi}_{0}(i)= & f(i)+\varepsilon_{0}(i), \\
\varepsilon_{0}(i)= & -\lambda_{0} L^{1 / 2}\left|\xi_{0}(i)-v_{a}(i)\right|^{1 / 2} \operatorname{sign}\left(\xi_{0}(i)-v_{a}(i)\right) \\
& +\xi_{1}(i) \\
\dot{\xi}_{1}(i)= & \varepsilon_{1}(i) \\
\varepsilon_{1}(i)= & -\lambda_{1} L \operatorname{sign}\left(\xi_{1}(i)-\varepsilon_{0}(i)\right),
\end{aligned}
$$

where $\xi_{0}(i)$ and $\xi_{1}(i)$ are the estimations of $v_{a}(i)$ and disturbance $d_{l}(i)$, respectively, $i=1,2, \cdots, 6 . \lambda_{0}, \lambda_{1}$ and $L$ are the coefficients of the FTDO to be selected. 
Considering the dynamics model (8) and FTDO (9), the error dynamics of the FTDO understood in the Filippov sense can be obtained by

$$
\begin{aligned}
& \dot{e}_{0}(i)=-\lambda_{0} L^{1 / 2}\left|e_{0}(i)\right|^{1 / 2} \operatorname{sign}\left(e_{0}(i)\right)+e_{1}(i), \\
& \dot{e}_{1}(i) \in-\lambda_{1} L \operatorname{sign}\left(e_{1}(i)-\dot{e}_{0}(i)\right)+[-L, L],
\end{aligned}
$$

where the estimation errors are defined by $e_{0}=\xi_{0}-v_{a}$ and $e_{1}=\xi_{1}-d_{l}$. It follows from $[31,32]$ that the estimation errors $e_{0}(t)$ and $e_{1}(t)$ will converge to zero in finite time, which implies that there exists a time constant $t_{f}>0$ such that $e_{0}(t)=0$ and $e_{1}(t)=0$ for $t>t_{f}$. Additionally, it is true that $\xi_{1}=\varepsilon_{0}$ for $t>t_{f}$.

3.2. Finite-Time Disturbance Observer Based Full-Order Terminal Sliding Mode Controller (FTDO-FOTSMC). Define the tracking errors of the manned submersible as

$$
\begin{aligned}
& e_{\eta}=\eta-\eta_{d}, \\
& e_{v}=v_{a}-\dot{\eta}_{d},
\end{aligned}
$$

where $\eta_{d}$ and $\dot{\eta}_{d}$ are the desired reference trajectory and its derivative, respectively.

The error dynamics of the manned submersible can be obtained by

$$
\begin{aligned}
& \dot{e}_{\eta}=e_{v}, \\
& \dot{e}_{v}=-\bar{C} v_{a}-\bar{D} v_{a}-\bar{G}+\bar{M} B u+d_{l}-\ddot{\eta}_{d} .
\end{aligned}
$$

The full-order terminal sliding surface is designed by

$$
\sigma=e_{v}+\int_{0}^{\tau}\left(\Lambda_{\eta} \frac{e_{\eta}}{\left\|e_{\eta}\right\|^{1-\alpha_{\eta}}}+\Lambda_{v} \frac{e_{v}}{\left\|e_{v}\right\|^{1-\alpha_{v}}}\right) d \tau,
$$

where $\Lambda_{\eta}=\operatorname{diag}\left\{\lambda_{\eta 1}, \cdots, \lambda_{\eta 6}\right\}$ and $\Lambda_{v}=\operatorname{diag}\left\{\lambda_{v 1}, \cdots, \lambda_{v 6}\right\}$ are the positive definite diagonal matrices, whose elements make $P_{i}(s)=s^{2}+\lambda_{v i} s+\lambda_{\eta i}(i=1, \cdots, 6)$ be Hurwitz polynomials. The parameters $\alpha_{\eta}$ and $\alpha_{v}$ satisfy the equation of $\alpha_{\eta}=\alpha_{v} \alpha /\left(2 \alpha-\alpha_{v}\right)$, where $\alpha \in(0,1)$.

Taking the time derivative of the full-order terminal sliding surface (13) along the error dynamics (12), we can obtain the sliding-mode dynamics as

$$
\begin{aligned}
\dot{\sigma}= & \dot{e}_{v}+\Lambda_{\eta} \frac{e_{\eta}}{\left\|e_{\eta}\right\|^{1-\alpha_{\eta}}}+\Lambda_{v} \frac{e_{v}}{\left\|e_{v}\right\|^{1-\alpha_{v}}} \\
= & -\bar{C} v_{a}-\bar{D} v_{a}-\bar{G}+\bar{M} B u+d_{l}-\ddot{\eta}_{d}+\Lambda_{\eta} \frac{e_{\eta}}{\left\|e_{\eta}\right\|^{1-\alpha_{\eta}}} \\
& +\Lambda_{v} \frac{e_{v}}{\left\|e_{v}\right\|^{1-\alpha_{v}}} .
\end{aligned}
$$

The continuous sliding-mode control law can be designed as

$$
\begin{aligned}
u & =(\bar{M} B)^{\dagger}\left(u_{e q u}+u_{s m c}\right), \\
u_{e q u} & =\bar{C} v_{a}+\bar{D} v_{a}+\bar{G}+\ddot{\eta}_{d}-\Lambda_{\eta} \frac{e_{\eta}}{\left\|e_{\eta}\right\|^{1-\alpha_{\eta}}}
\end{aligned}
$$

$$
\begin{gathered}
-\Lambda_{v} \frac{e_{v}}{\left\|e_{v}\right\|^{1-\alpha_{v}}}-\xi_{1}, \\
u_{s m c}=-k_{1} \sigma-k_{2} \frac{\sigma}{\|\sigma\|^{1-\beta}},
\end{gathered}
$$

where $(\bar{M} B)^{\dagger}=(\bar{M} B)^{T}\left[(\bar{M} B)(\bar{M} B)^{T}\right]^{-1}$ represents the pseudo-inverse. $k_{1}>0, k_{2}>0$ and $0<\beta<1$ are the controller parameters to be selected.

\section{Stability Analysis}

The stability analysis of the closed-loop manned submersible system is given in this section.

Theorem 1. For the manned submersible system (12) with the full-order terminal sliding surface (13) under the continuous sliding-mode control law (15), the tracking error $e_{\eta}$ of the manned submersible will converge to the origin in finite time despite the presence of lumped disturbances.

Proof. In the first step, we will show that the bounded estimation errors $e_{1}$ will not make the sliding variable $\sigma$ go to infinity in finite time.

Substituting the sliding-mode control law (15) into the sliding-mode dynamics (14), we can obtain the closed-loop sliding-mode dynamics as follows:

$$
\dot{\sigma}=-k_{1} \sigma-k_{2} \frac{\sigma}{\|\sigma\|^{1-\beta}}+e_{1},
$$

where $e_{1}=\xi_{1}-d_{l}$ is estimation error of the FTDO. Furthermore, according to the Section 3.1, we can obtain that the estimation error $e_{1}$ will converge to zero in finite time $t_{f}>0$.

Define a finite-time bounded (FTB) function [33] for (16) as

$$
V_{1}(\sigma)=\frac{1}{2} \sigma^{T} \sigma
$$

Taking the time derivative of the FTB function (17) along the dynamics (16), we can obtain the following:

$$
\begin{aligned}
\dot{V}_{1}(\sigma) & =\sigma^{T} \dot{\sigma} \\
& =\sigma^{T}\left(-k_{1} \sigma-k_{2} \frac{\sigma}{\|\sigma\|^{1-\beta}}+e_{1}\right) \\
& =-k_{1}\|\sigma\|^{2}-k_{2}\|\sigma\|^{1+\beta}+\sigma^{T} e_{1} \\
& \leq \sigma^{T} e_{1} \\
& \leq \frac{1}{2}\left(\|\sigma\|^{2}+\left\|e_{1}\right\|^{2}\right) \\
& \leq K_{v 1} V_{1}+L_{v 1},
\end{aligned}
$$

where $K_{v 1}=1$ and $L_{v 1}=(1 / 2)\left\|e_{1}\right\|^{2}$.

Therefore, it can be acquired from (18) that $V_{1}(\sigma)$ and so $\sigma$ will not escape to infinity in any finite time. 
In the second step, we will show that the bounded sliding variable $\sigma$ will not drive $e_{\eta}$ and $e_{v}$ to infinity in finite time.

According to the manned submersible system (12) and the full-order terminal sliding surface (13), we can obtain the error dynamics as follows:

$$
\begin{aligned}
& \dot{e}_{\eta}=e_{v}, \\
& \dot{e}_{v}=-\Lambda_{\eta} \frac{e_{\eta}}{\left\|e_{\eta}\right\|^{1-\alpha_{\eta}}}-\Lambda_{v} \frac{e_{v}}{\left\|e_{v}\right\|^{1-\alpha_{v}}}+\dot{\sigma} .
\end{aligned}
$$

Define a finite-time bounded (FTB) function for (19) as

$$
V_{2}\left(e_{\eta}, e_{v}\right)=\frac{1}{2} e_{\eta}^{T} e_{\eta}+\frac{1}{2} e_{v}^{T} e_{v}
$$

Taking the time derivative of the FTB function (20) along the dynamics (19), and using the inequality $\|x\|^{\rho}<1+\|x\|$ for $\rho \in(0,1)$, we can obtain the following:

$$
\begin{aligned}
& \dot{V}_{2}\left(e_{\eta}, e_{v}\right)=\frac{1}{2} e_{\eta}^{T} e_{\eta}+\frac{1}{2} e_{v}^{T} e_{v}=e_{\eta}^{T} \dot{e}_{\eta}+e_{v}^{T} \dot{e}_{v}=e_{\eta}^{T} e_{v} \\
& \quad+e_{v}^{T}\left(-\Lambda_{\eta} \frac{e_{\eta}}{\left\|e_{\eta}\right\|^{1-\alpha_{\eta}}}-\Lambda_{v} \frac{e_{v}}{\left\|e_{v}\right\|^{1-\alpha_{v}}}+\dot{\sigma}\right) \\
& \leq\left\|e_{\eta}\right\|\left\|e_{v}\right\|+\left\|e_{v}\right\|\left(\gamma_{\eta}\left\|e_{\eta}\right\|^{\alpha_{\eta}}+\gamma_{v}\left\|e_{v}\right\|^{\alpha_{v}}+k_{1}\|\sigma\|\right. \\
&\left.+k_{2}\|\sigma\|^{\beta}+\left\|e_{1}\right\|\right) \\
& \leq\left\|e_{\eta}\right\|\left\|e_{v}\right\|+\left\|e_{v}\right\|\left(\gamma_{\eta}\left(1+\left\|e_{\eta}\right\|\right)+\gamma_{v}\left(1+\left\|e_{v}\right\|\right)\right. \\
&\left.+k_{1}\|\sigma\|+k_{2}(1+\|\sigma\|)+\left\|e_{1}\right\|\right) \\
& \leq \frac{\left\|e_{\eta}\right\|^{2}+\left\|e_{v}\right\|^{2}}{2}+\gamma_{\eta}\left(\frac{\left\|e_{v}\right\|^{2}+1}{2}+\frac{\left\|e_{v}\right\|^{2}+\left\|e_{\eta}\right\|^{2}}{2}\right) \\
& \leq K_{v 2} V_{2}+L_{v 2}, \\
&\left.+\frac{1}{2}+\frac{\gamma_{\eta}}{2}\right)\left\|e_{\eta}\right\|^{2}+\left(1+\gamma_{\eta}+\frac{3 \gamma_{v}}{2}+\frac{k_{1}}{2}+k_{2}+\left\|e_{1}\right\|^{2}\right. \\
&+ k_{1} \frac{\left\|e_{v}\right\|^{2}+\|\sigma\|^{2}}{2}+k_{2}\left(\frac{\left\|e_{v}\right\|^{2}+1}{2}+\frac{\left\|e_{v}\right\|^{2}+\|\sigma\|^{2}}{2}\right)
\end{aligned}
$$

where $K_{v 2}=2+2 \gamma_{\eta}+3 \gamma_{v}+k_{1}+2 k_{2}$ and $L_{v 2}=\gamma_{\eta} / 2+\gamma_{v} / 2+$ $\left(k_{1} / 2\right)\|\sigma\|^{2}+\left(k_{2} / 2\right)\left(1+\|\sigma\|^{2}\right)+(1 / 2)\left\|e_{1}\right\|^{2} \cdot \gamma_{\eta}$ and $\gamma_{v}$ denote the maximum eigenvalue of the matrices $\Lambda_{\eta}$ and $\Lambda_{v}$, respectively.

Therefore, we can obtain that $V_{2}\left(e_{\eta}, e_{v}\right)$ and so $e_{\eta}, e_{v}$ will not escape to infinity in any finite time.
In the third step, we will show that the sliding variable $\sigma$ will converge to the desired sliding surface $\sigma=0$ in finite time.

Since the estimation error $e_{1}$ of FTDO will converge to zero in finite time, the sliding-mode dynamics (16) will reduce to

$$
\dot{\sigma}=-k_{1} \sigma-k_{2} \frac{\sigma}{\|\sigma\|^{1-\beta}} .
$$

It follows from [34] that the sliding variable $\sigma$ is able to converge to the desired sliding surface $\sigma=0$ in finite time.

In the fourth step, we will show that the tracking error $e_{\eta}$ of the manned submersible will converge to the origin in finite time.

Once the sliding surface $\sigma=0$ is achieved in finite time, the system dynamics (19) will reduce to

$$
\begin{aligned}
& \dot{e}_{\eta}=e_{v}, \\
& \dot{e}_{v}=-\Lambda_{\eta} \frac{e_{\eta}}{\left\|e_{\eta}\right\|^{1-\alpha_{\eta}}}-\Lambda_{v} \frac{e_{v}}{\left\|e_{v}\right\|^{1-\alpha_{v}}} .
\end{aligned}
$$

It can be obtained from [34] that system (23) is finite-time stable, which means that the tracking error $e_{\eta}$ will converge to zero in finite time.

In summary, the position and Euler angle of the manned submersible is able to track the desired trajectory in finite time despite the presence of lumped disturbances.

\section{Simulation Results}

This section presents some numerical simulation results to demonstrate the effectiveness of the proposed finite-time disturbance observer based full-order terminal sliding-mode controller (FTDO-FOTSMC) of the manned submersible. Additionally, in order to verify the superiority of the proposed control method, both the conventional full-order terminal sliding-mode control (FOTSMC) method [30] and nonlinear disturbance observer based sliding-mode control (NDOSMC) method [26] are employed as comparative methods.

The parameters of the controllers are given as follows. The coefficients of the FTDO are $\lambda_{0}=3, \lambda_{1}=3$ and $L=12$. The coefficients of the full-order terminal sliding surface are $\Lambda_{\eta}=$ $\operatorname{diag}(6, \cdots, 6), \Lambda_{v}=\operatorname{diag}(9, \cdots, 9), \alpha_{\eta}=2 / 3$ and $\alpha_{v}=4 / 5$. The control gains are $k_{1}=1, k_{2}=0.5$ and $\beta=0.5$.

The lumped disturbances applied to the manned submersible system are given by $d_{l}=\bar{M}(\eta, v) d_{s}=\bar{M}(\eta, v)\left(d_{\Delta}+\right.$ $d)$, where $d_{\Delta}$ represents the model uncertainties $d_{\Delta}=$ $-\Delta M \dot{v}-\Delta C(v) v-\Delta D(v) v-\Delta G(\eta)$ and $d$ represents the external disturbances $d=\sum_{i=1}^{N}\left[A_{i 1} \cos \left(\omega_{i} t+\alpha_{i}\right)+A_{i 2} \sin \left(\omega_{i} t+\right.\right.$ $\left.\alpha_{i}\right)$ ] [35].

All the initial values of the state variables of the manned submersible are set to be zero, except for the position $z_{0}=$ $-4 \mathrm{~m}$. 

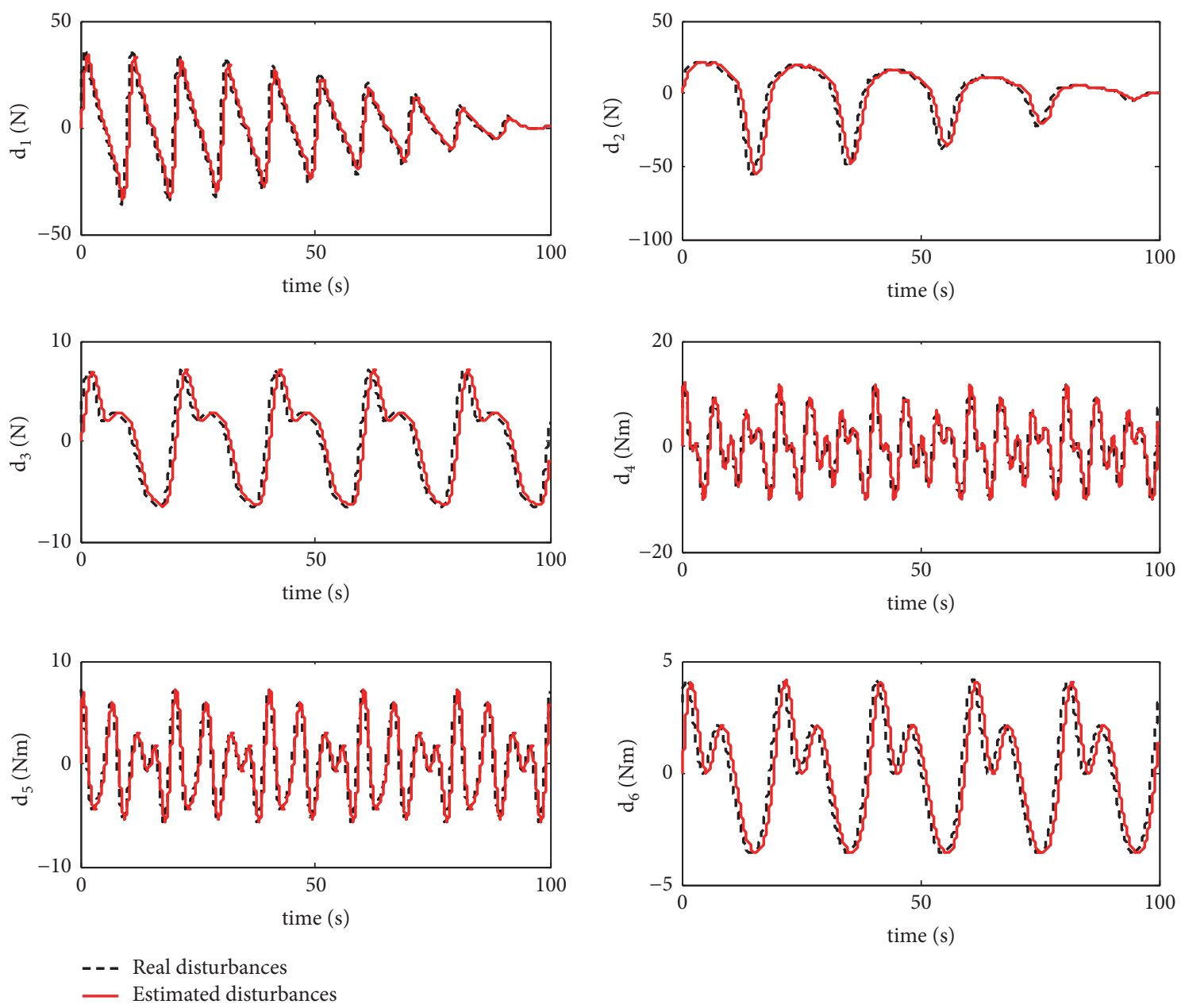

FIGURE 3: Disturbances estimation.

The desired reference trajectory is given by

$$
\begin{aligned}
& x_{r}= \begin{cases}0 & t \leq 25 s, \\
t-25 & 25<t \leq 50 s, \\
25 & 50<t \leq 75 s, \\
-t+100 & t>75 s,\end{cases} \\
& y_{r}= \begin{cases}t & t \leq 25 s, \\
25 & 25<t \leq 50 s, \\
-t+75 & 50<t \leq 75 s, \\
0 & t>75 s,\end{cases} \\
& z_{r}=-4-0.01 t \quad t>0 s, \\
& \varphi_{r}=0.1 \sin (0.3 t) \quad t>0 s, \\
& \theta_{r}=-0.05 \sin (0.3 t) \quad t>0 s,
\end{aligned}
$$

The manned submersible is required to track a square reference trajectory in the X-Y plane. Meanwhile, it descends from the depth of $4 \mathrm{~m}$ to $5 \mathrm{~m}$ in the vertical direction. Additionally, the Euler angles of the manned submersible should track a time-varying trajectory. This comprehensive reference trajectory mainly examines the forward and sideslip control performances, as well as to verify the existence of the cross-couplings among pitch, roll and yaw axes.

The simulation results are shown in Figures 3-8. Figure 3 depicts the estimations of the lumped disturbances by FTDO. It can be observed that the FTDO is able to estimate the disturbances accurately and timely, which is the basis of compensating the lumped disturbances. Figures 4 and 5 illustrate the response curves of position and Euler angles, respectively. We can see that the decent tracking performances can be obtained in all six channels under the proposed FTDOFOTSMC method. The position and Euler angles of the manned submersible under the conventional FOTSMC are almost able to track the reference trajectory. However, it suffers from fluctuations to some degree. Additionally, the position and Euler angles of the manned submersible under the NDO-SMC are able to track the reference trajectory. Its tracking accuracy is better than FOTSMC method, but worse 

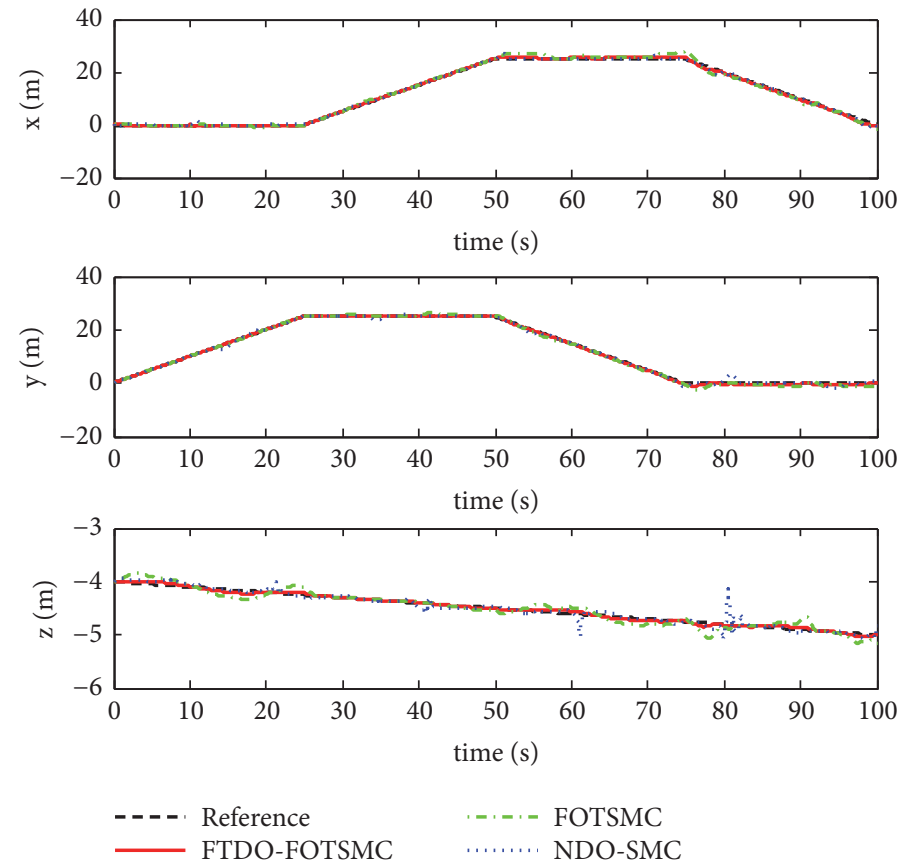

FIGURE 4: Response curves of position.
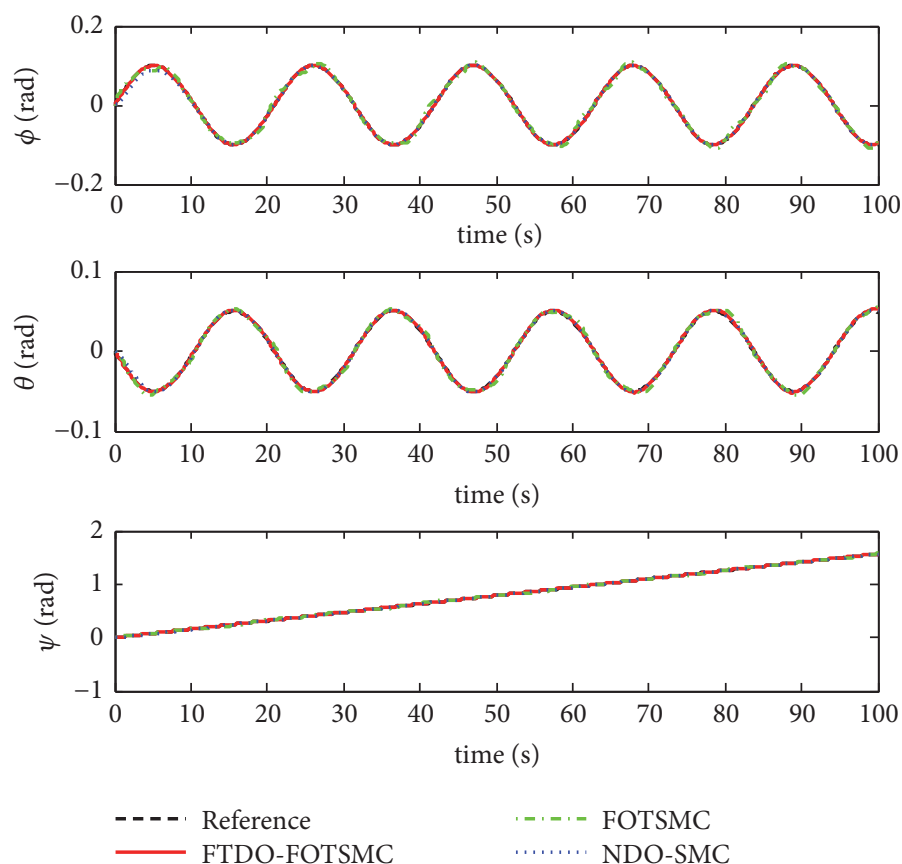

FIgURE 5: Response curves of Euler angles.

than FTDO-FOTSMC method. Moreover, to stand out the superiority of the proposed FTDO-FOTSMC method, the response curves of tracking errors under three control methods are given in Figure 6. The tracking errors of the manned submersible under the proposed FTDO-FOTSMC method are smaller than the conventional FOTSMC method and
NDO-SMC method, which implies that the proposed FTDOFOTSMC method outperforms the conventional FOTSMC method and NDO-SMC method for its much more accurate tracking performance and milder transient process. Furthermore, the tracking performance of the manned submersible in $\mathrm{X}-\mathrm{Y}$ plane is depicted in Figure 7. It can be acquired that the 

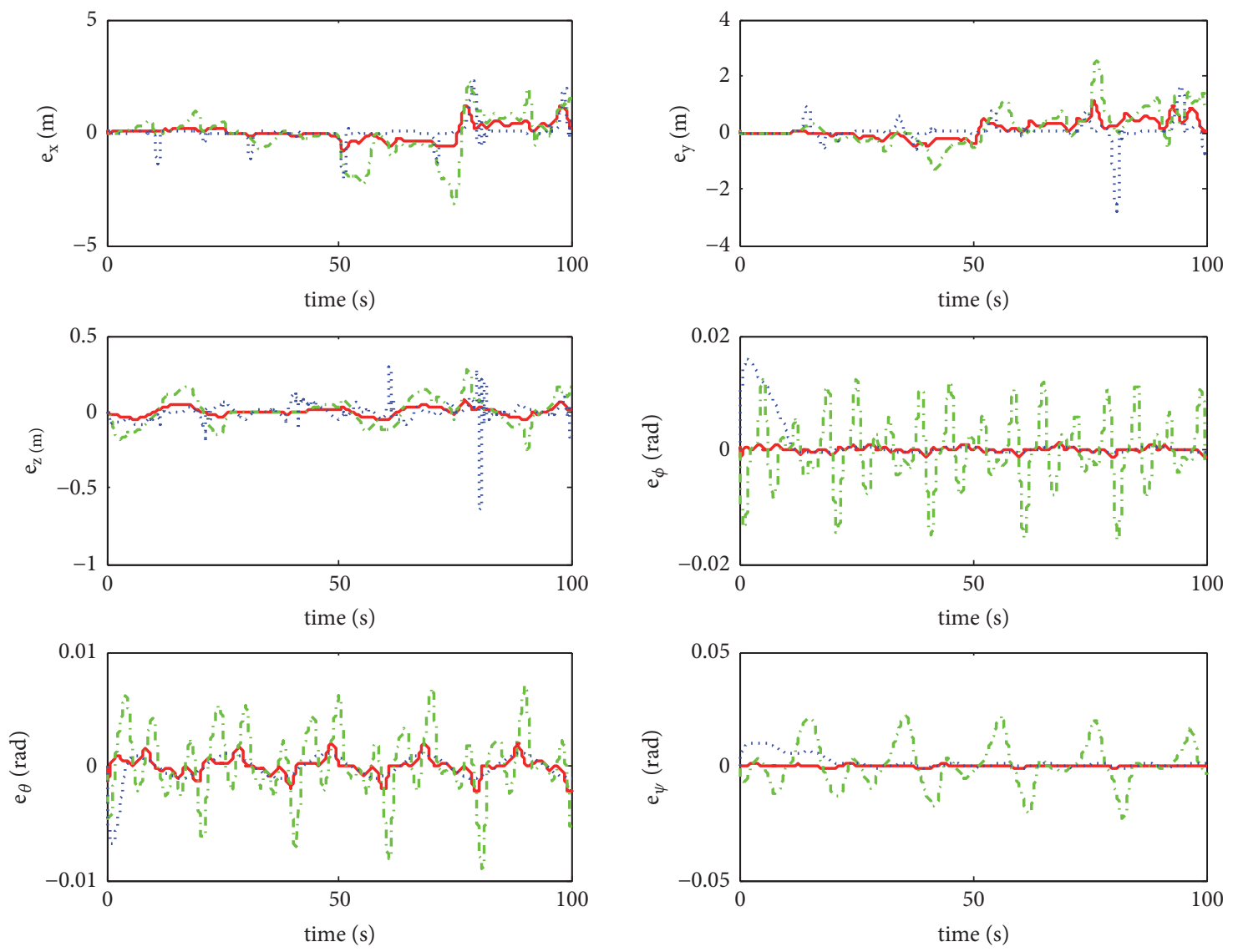

- FTDO-FOTSMC

- - FOTSMC

NDO-SMC

FIGURE 6: Response curves of tracking errors.
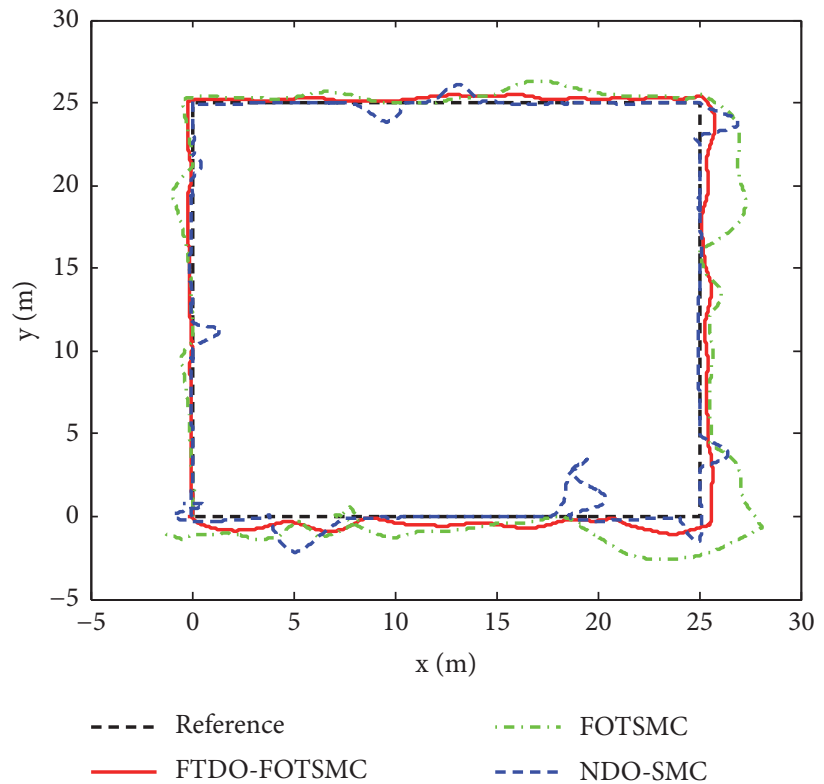

FIgURE 7: Response curves of position in X-Y plane. 

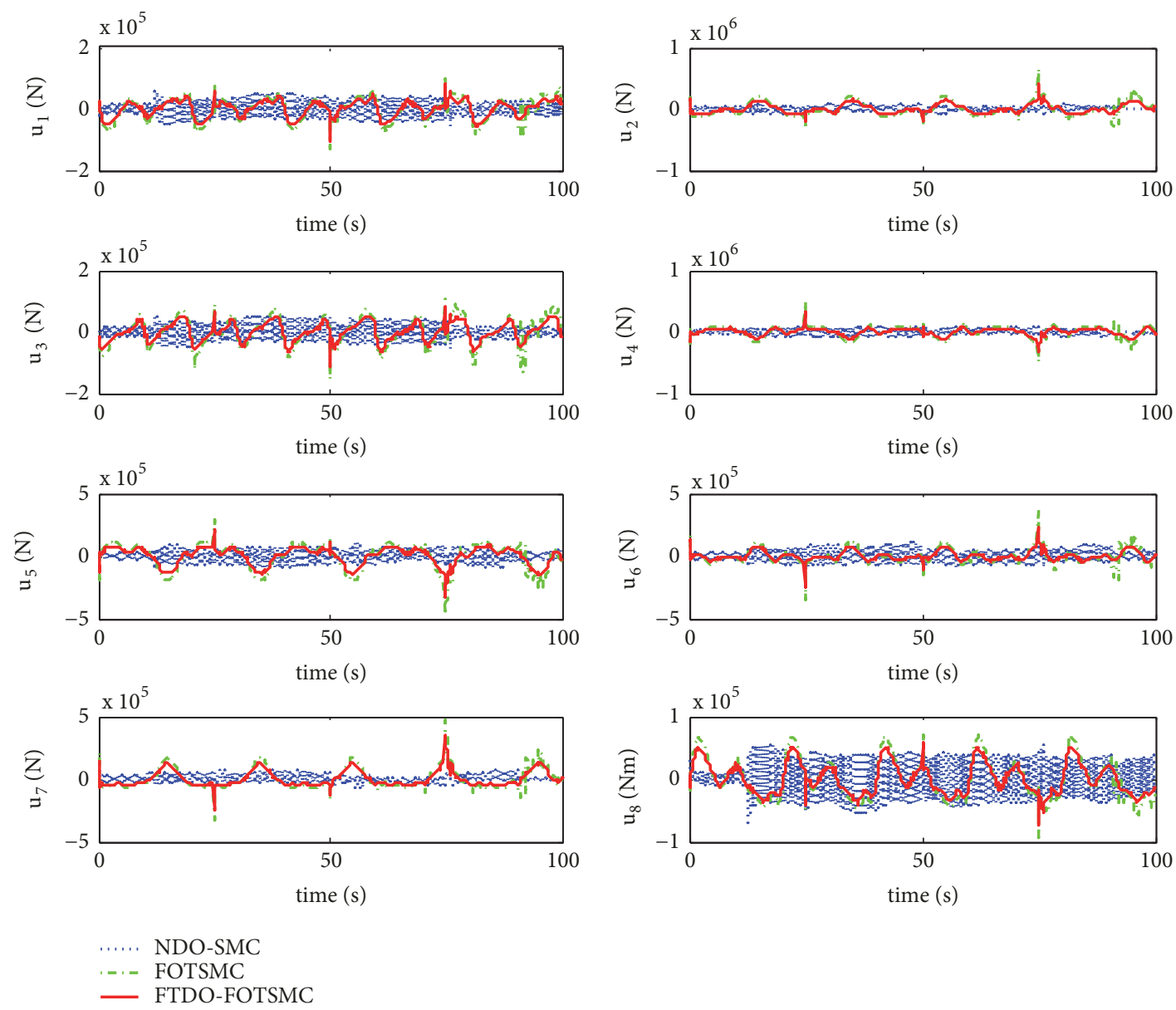

FIGURE 8: Response curves of control inputs.

TABLE 1: The root-mean-square (RMS) of tracking errors.

\begin{tabular}{lcccccc}
\hline & $\left(e_{x}\right)_{R M S}$ & $\left(e_{y}\right)_{R M S}$ & $\left(e_{z}\right)_{R M S}$ & $\left(e_{\varphi}\right)_{R M S}$ & $\left(e_{\theta}\right)_{R M S}$ & $\left(e_{\psi}\right)_{R M S}$ \\
\hline FTDO-FOTSMC & $0.3591 \mathrm{~m}$ & $0.3289 \mathrm{~m}$ & $0.0277 \mathrm{~m}$ & $0.0005 \mathrm{rad}$ & $0.0008 \mathrm{rad}$ & $0.0004 \mathrm{rad}$ \\
FOTSMC & $0.9082 \mathrm{~m}$ & $0.6322 \mathrm{~m}$ & $0.0920 \mathrm{~m}$ & $0.0061 \mathrm{rad}$ & $0.0030 \mathrm{rad}$ & $0.0097 \mathrm{rad}$ \\
NDO-SMC & $0.4170 \mathrm{~m}$ & $0.4479 \mathrm{~m}$ & $0.0612 \mathrm{~m}$ & $0.0036 \mathrm{rad}$ & $0.0012 \mathrm{rad}$ & $0.0033 \mathrm{rad}$ \\
\hline
\end{tabular}

manned submersible is able to perform the square trajectory precisely under the proposed FTDO-FOTSMC method. On the other hand, both the conventional FOTSMC method and NDO-SMC method drive the manned submersible to track the square trajectory with relatively large errors. Finally, the response curves of control inputs are shown in Figure 8. With the continuous control law of the proposed FTDO-FOTSMC method and FOTSMC method, the chattering phenomenon can be alleviated greatly or even eliminated completely. However, the NDO-SMC method is subject to chattering phenomenon to a certain degree for its discontinuous control action.

To further demonstrate the superiority of the proposed FTDO-FOTSMC method, the tracking performance is compared quantitatively. The root-mean-square (RMS) that is a common criterion to measure the tracking performance is given in Table 1. As reported in Table 1, the proposed FTDO-FOTSMC method attains the smaller RMS values than the conventional FOTSMC method and NDO-SMC method in all six channels, which reveals that the proposed FTDO-FOTSMC method is able to deal with the external disturbances and model uncertainties, as well as to achieve accurate tracking performance.

\section{Conclusions}

A novel finite-time disturbance observer based full-order terminal sliding mode control (FTDO-FOTSMC) method is proposed for the manned submersible with external disturbance and model uncertainties. With the proposed control method, the ideal sliding-mode motion of the manned 
submersible system behaves as desirable full-order dynamics. Furthermore, the outputs of the manned submersible system are able to converge to the desired reference trajectory in finite time. Finally, both the stability analysis and simulation results demonstrate the effectiveness of the proposed FTDOFOTSMC method for the manned submersible system. The future work will focus on the experimental tests on the manned submersible system. Additionally, the applications of the FTDO-FOTSMC method on unmanned underwater vehicles is another important work in future.

\section{Data Availability}

The data used to support the findings of this study are included within the article.

\section{Conflicts of Interest}

The authors declare that they have no conflicts of interest.

\section{Acknowledgments}

This work is supported by the National Natural Science Foundation of China under grants 61803182, 61703118, and the Natural Science Foundation of Jiangsu Province under grants BK20180593, BK20180592.

\section{References}

[1] W. Kohnen, "2007 MTS overview of manned underwater vehicle activity," Marine Technology Society Journal, vol. 42, no. 1, pp. 26-37, 2008.

[2] F. Liu, W. Cui, and X. Li, "China's first deep manned submersible, JIAOLONG," Science China Earth Sciences, vol. 53, no. 10, pp. 1407-1410, 2010.

[3] D. Zhu, Q. Liu, and Z. Hu, "Fault-tolerant control algorithm of the manned submarine with multi-thruster based on quantumbehaved particle swarm optimisation," International Journal of Control, vol. 84, no. 11, pp. 1817-1829, 2011.

[4] E. Zakeri, S. Farahat, S. A. Moezi, and A. Zare, "Path planning for unmanned underwater vehicle in $3 \mathrm{D}$ space with obstacles using spline-imperialist competitive algorithm and optimal interval type-2 fuzzy logic controller," Latin American Journal of Solids and Structures, vol. 13, no. 6, pp. 1054-1085, 2016.

[5] E. Zakeri, S. Farahat, S. A. Moezi, and M. Zare, "Optimal robust control of an unmanned underwater vehicle independent of hydrodynamic forces using firefly optimization algorithm," in Proceedings of the 24th Annual International Conference on Mechanical Engineering, pp. 1-6, 2016.

[6] T. I. Fossen, Guidance and control of ocean vehicles, Wiley, New York, NY, USA, 1994.

[7] J. Y. Xie, W. B. Xue, H. Zhang, P. F. Xu, and W. C. Cui, "Dynamic modeling and investigation of maneuver characteristics of a deep-sea manned submarine vehicle," China Ocean Engineering, vol. 23, no. 3, pp. 505-516, 2009.

[8] L. Moreira and C. Guedes Soares, "H2 and Hinfin; designs for diving and course control of an autonomous underwater vehicle in presence of waves," IEEE Journal of Oceanic Engineering, vol. 33 , no. 2, pp. 69-88, 2008.
[9] H. Wang, K. Liu, and S. Li, "Command filter based globally stable adaptive neural control for cooperative path following of multiple underactuated autonomous underwater vehicles with partial knowledge of the reference speed," Neurocomputing, vol. 27, no. 5, pp. 1478-1489, 2018.

[10] B. Sun, D. Zhu, and S. X. Yang, "A bioinspired filtered backstepping tracking control of 7000-m manned submarine vehicle," IEEE Transactions on Industrial Electronics, vol. 61, no. 7, pp. 3682-3693, 2014.

[11] R. Yu, Q. Zhu, G. Xia, and Z. Liu, "Sliding mode tracking control of an underactuated surface vessel," IET Control Theory \& Applications, vol. 6, no. 3, pp. 461-466, 2012.

[12] E. Zakeri, S. Farahat, S. A. Moezi, and A. Zare, "Robust sliding mode control of a mini unmanned underwater vehicle equipped with a new arrangement of water jet propulsions: Simulation and experimental study," Applied Ocean Research, vol. 59, pp. 521-542, 2016.

[13] M. T. Alrifai and M. Zribi, "Sliding Mode Control of Chaos in a Single Machine Connected to an Infinite Bus Power System," Mathematical Problems in Engineering, vol. 2018, Article ID 2703684, 13 pages, 2018.

[14] A. Šabanovic, "Variable structure systems with sliding modes in motion control-a survey," IEEE Transactions on Industrial Informatics, vol. 7, no. 2, pp. 212-223, 2011.

[15] E. Zakeri, S. A. Moezi, and M. Eghtesad, "Tracking control of ball on sphere system using tuned fuzzy sliding mode controller based on artificial bee colony algorithm," International Journal of Fuzzy Systems, vol. 20, no. 1, pp. 295-308, 2018.

[16] A. Levant, "Principles of 2-sliding mode design," Automatica, vol. 43, no. 4, pp. 576-586, 2007.

[17] V. I. Utkin, Sliding Modes in Control Optimization, Springer, Berlin, Germany, 1992.

[18] S. H. Li, J. Yang, W. H. Chen, and X. Chen, Disturbance observer based control: methods and applications, USA, Prentice-Hall, 2014.

[19] J. Yang, J. Su, S. Li, and X. Yu, "High-order mismatched disturbance compensation for motion control systems via a continuous dynamic sliding-mode approach," IEEE Transactions on Industrial Informatics, vol. 10, no. 1, pp. 604-614, 2014.

[20] B. Xu and F. C. Sun, "Composite intelligent learning control of strict-feedback systems with disturbance," IEEE Transactions on Cybernetics, vol. 48, no. 2, pp. 730-741, 2018.

[21] Z. Gao, "On the centrality of disturbance rejection in automatic control," ISA Transactions, vol. 53, no. 4, pp. 850-857, 2014.

[22] W. H. Chen, J. Yang, L. Guo, and S. H. Li, "Disturbanceobserver-based control and related methods-an overview," IEEE Transactions on Industrial Electronics, vol. 63, no. 2, pp. 10831095, 2016.

[23] J. Yang, W.-H. Chen, S. Li, L. Guo, and Y. Yan, "Disturbance/Uncertainty Estimation and Attenuation Techniques in PMSM Drives - A Survey," IEEE Transactions on Industrial Electronics, vol. 64, no. 4, pp. 3273-3285, 2017.

[24] X. Fang, A. Wu, Y. Shang, and C. Du, "Multivariable Super Twisting Based Robust Trajectory Tracking Control for Small Unmanned Helicopter," Mathematical Problems in Engineering, vol. 2015, Article ID 620357, 13 pages, 2015.

[25] L. Sun, Q. Hua, D. Li, L. Pan, Y. Xue, and K. Y. Lee, “Direct energy balance based active disturbance rejection control for coal-fired power plant," ISA Transactions, vol. 70, pp. 486-493, 2017. 
[26] J. Yang, S. Li, and X. Yu, "Sliding-mode control for systems with mismatched uncertainties via a disturbance observer," IEEE Transactions on Industrial Electronics, vol. 60, no. 1, pp. 160-169, 2013.

[27] X. Wei and L. Guo, "Composite disturbance-observer-based control and terminal sliding mode control for non-linear systems with disturbances," International Journal of Control, vol. 82, no. 6, pp. 1082-1098, 2009.

[28] X. Fang, A. Wu, Y. Shang, and N. Dong, "A novel sliding mode controller for small-scale unmanned helicopters with mismatched disturbance," Nonlinear Dynamics, vol. 83, no. 1-2, pp. 1053-1068, 2016.

[29] R. Cui, L. Chen, C. Yang, and M. Chen, "Extended State Observer-Based Integral Sliding Mode Control for an Underwater Robot With Unknown Disturbances and Uncertain Nonlinearities," IEEE Transactions on Industrial Electronics, vol. 64, no. 8, pp. 6785-6795, 2017.

[30] Y. Feng, F. Han, and X. Yu, "Chattering free full-order slidingmode control," Automatica, vol. 50, no. 4, pp. 1310-1314, 2014.

[31] A. Levant, "Higher-order sliding modes, differentiation and output-feedback control," International Journal of Control, vol. 76, no. 9-10, pp. 924-941, 2003.

[32] Y. B. Shtessel, I. A. Shkolnikov, and A. Levant, "Smooth secondorder sliding modes: missile guidance application," Automatica, vol. 43, no. 8, pp. 1470-1476, 2007.

[33] S. Li and Y. Tian, "Finite-time stability of cascaded time-varying systems," International Journal of Control, vol. 80, no. 4, pp. 646657, 2007.

[34] S. P. Bhat and D. S. Bernstein, "Geometric homogeneity with applications to finite-time stability," Mathematics of Control, Signals, and Systems, vol. 17, no. 2, pp. 101-127, 2005.

[35] Z. Liu, "Ship Adaptive Course Keeping Control with Nonlinear Disturbance Observer, IEEE Access, vol. 5, pp. 17567-17575, 2017. 


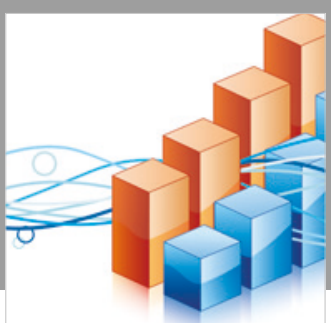

Advances in

Operations Research

\section{-n-m}
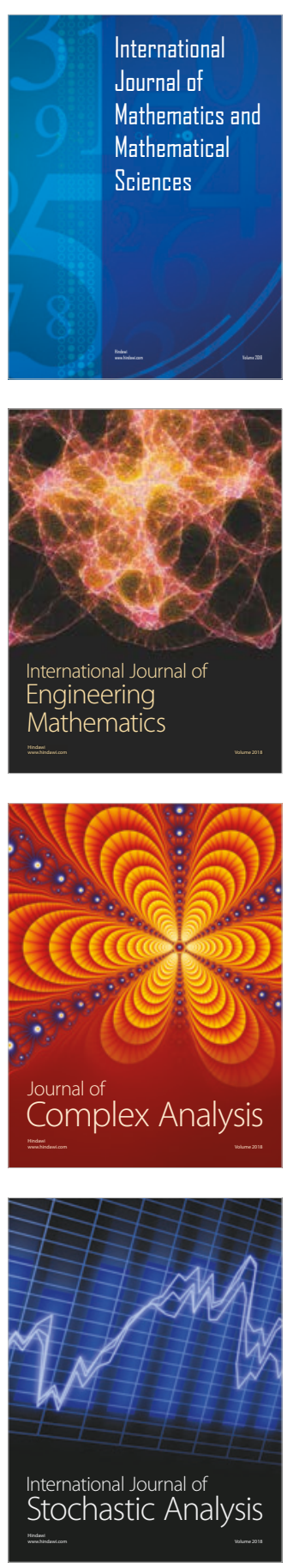
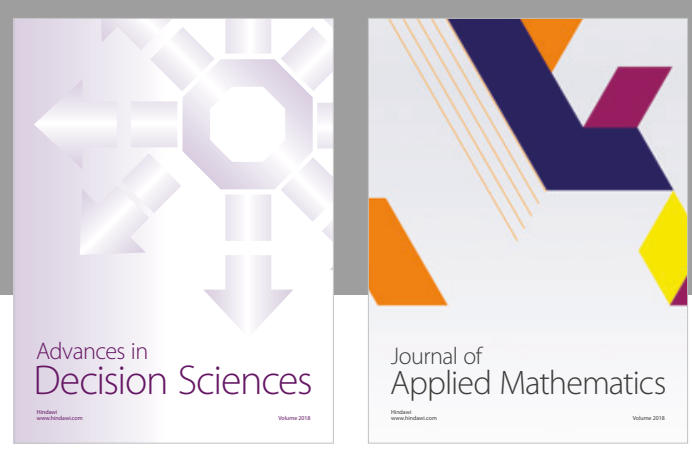

Journal of

Applied Mathematics
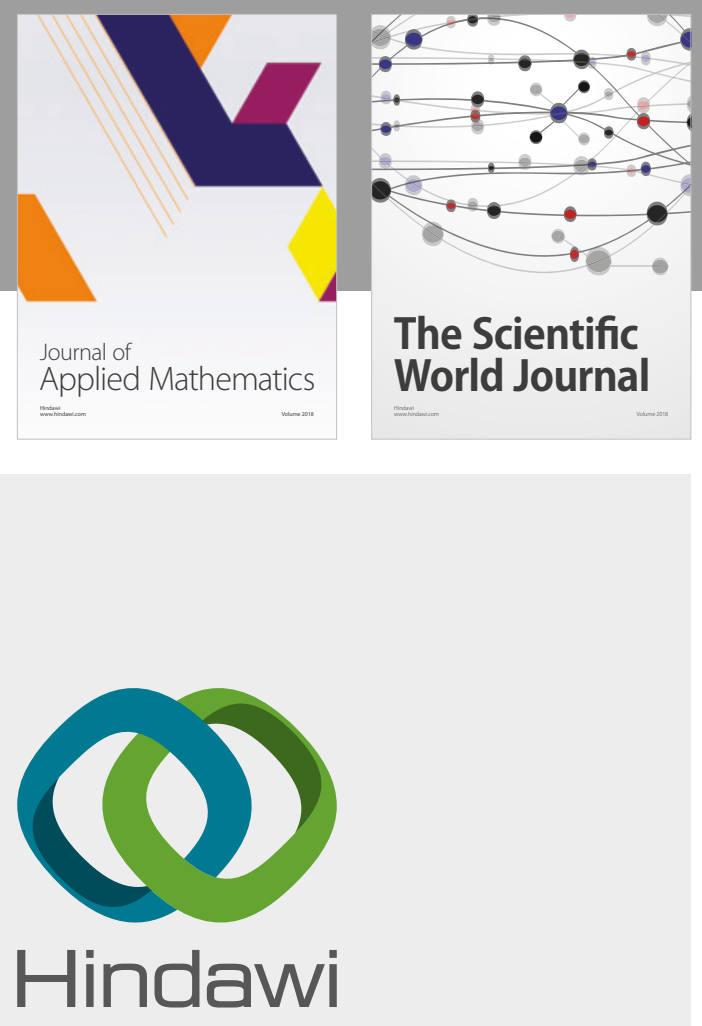

Submit your manuscripts at

www.hindawi.com

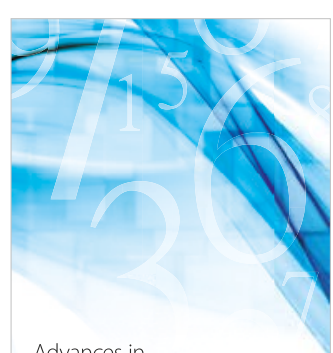

Advances in
Numerical Analysis
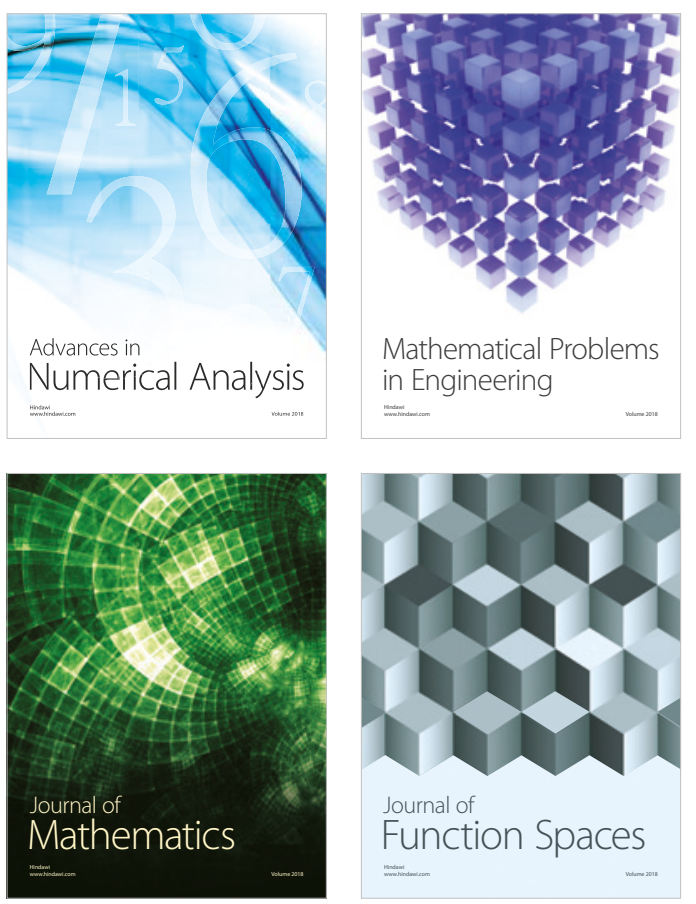

Mathematical Problems in Engineering

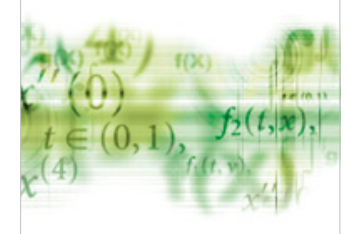

International Journal of

Differential Equations

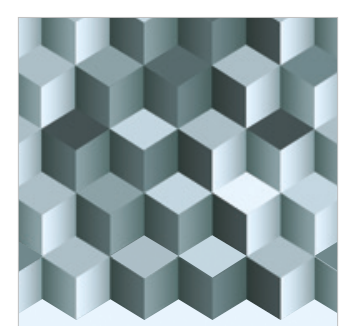

Journal of

Function Spaces

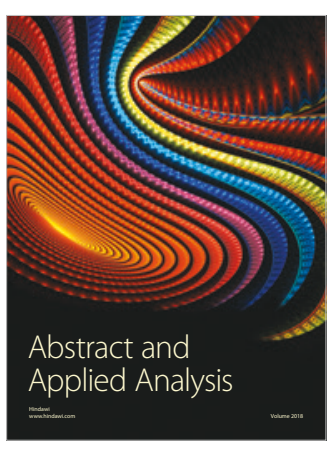

The Scientific

World Journal

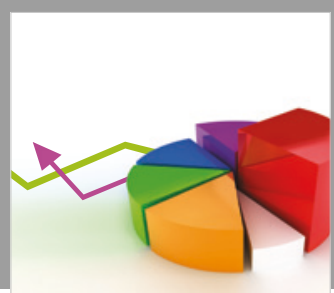

Journal of

Probability and Statistics
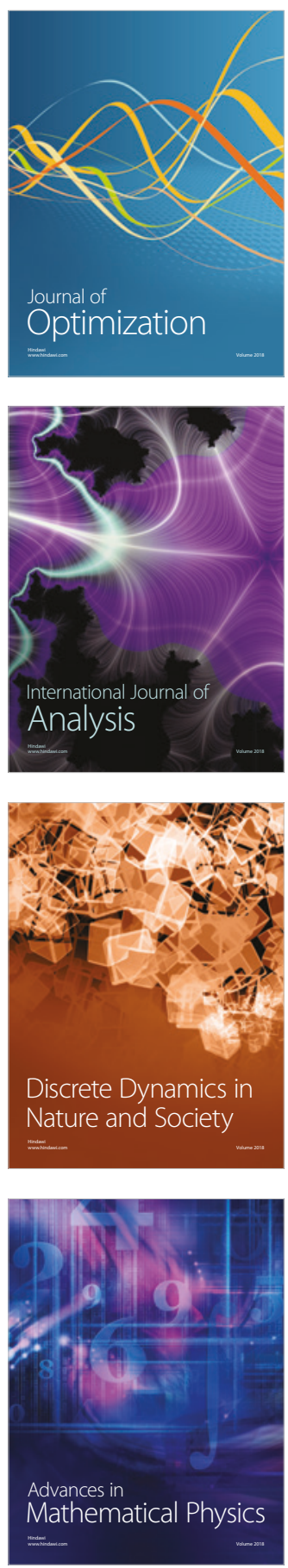